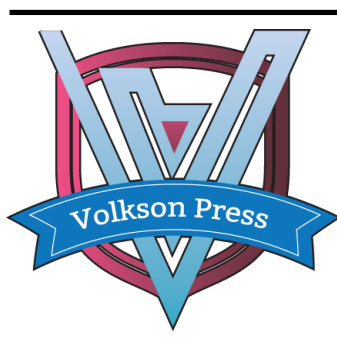

Contents List available at VOLKSON PRESS

New Materials and Intelligent Manufacturing (NMIM)

DOI : http://doi.org/10.26480/icnmim.01.2018.43.45

Journal Homepage: https://topicsonchemeng.org.my/

ISBN: 978-1-948012-12-6

\title{
EXTENSION OF VIKOR METHOD FOR SUPPLIER SELECTION BASED ON AXIOMATIC FUZZY SET
}

\author{
Lili Tao*, Lishi Zhang, Lifeng Zhang \\ School of Sciences, Dalian Ocean University, Heishijiao Street, Dalian, China
${ }^{*}$ Corresponding Author email: taolili1986@163.com
}

This is an open access article distributed under the Creative Commons Attribution License, which permits unrestricted use, distribution, and reproduction in any medium, provided the original work is properly cited

\section{ARTICLE DETAILS}

Article History:

Received 26 June 2018

Accepted 2 July 2018

Available online 1 August 2018

\section{ABSTRACT}

A new method is proposed to solve multi-criteria decision-making problems in which both the criteria values and criteria weights take the form of linguistic information based on the traditional idea of VIKOR method. In this paper, the linguistic values are represented by membership degrees applying the axiomatic fuzzy set (AFS) theory, so that the application of VIKOR method is extended. Moreover, the supplier selection is used to illustrate the application of the proposed approach.

\section{KEYWORDS}

Multi-criteria decision making, extended VIKOR method, AFS theory, supplier selection

\section{INTRODUCTION}

The VIKOR method was developed as a multi-criteria decision-making method to solve a discrete decision problem with non-commensurable and conflicting criteria. This method focuses on ranking and selecting from a set of alternatives and determines compromise solutions for a problem with conflicting criteria, which can help the decision makers to reach a final decision. Here, the compromise solution is a feasible solution which is the closest to the ideal, and a compromise means an agreement established by mutual concessions.

In classical VIKOR method, the performance ratings and the weights of the criteria are known precisely and given as exact values, whereas in the real world, because of incomplete or non-obtainable information, the criteria values and criteria weights are often not so deterministic, which usually have various types of linguistic variables or fuzzy terms. For instance, when evaluating the "rescue capacity" or "recovering capacity" of an emergency alternative, terms like "good", "medium", "poor" are usually used, and evaluating an emergency alternative's responding time, terms like "very long", "long", "short" can be used instead of numeric values. Thus, in such situations, the use of linguistic approach is necessary.

The available approaches for dealing with linguistic terms can be classified into three categories: (1) The extension principle; (2) The symbolic method; and (3) The 2-tuple fuzzy linguistic representation model. Despite the applicability of the former approaches to MCDM problems, which require to determine the fuzzy numbers. In the past few years, in order to solve above issue, numerous attempts have been carried out [1-3]. However, we find that the different choices of the fuzzy numbers and their distance functions can lead to different decision results. In addition, the exact values must be converted to fuzzy numbers in those approaches that will seriously affect the veracity of the decision results.

The purpose of this paper is to extend the method of VIKOR to develop a new methodology for solving MCDM problems under linguistic environment, i.e., the information about criteria weights and the criteria values take the form of exact values, linguistic variables or fuzzy terms. In this study, the AFS theory is applied to extend the approach of VIKOR for multi-type criteria. The AFS framework studies on how to convert the information in the dataset into the membership functions and logic operations of fuzzy concepts are determined according to the ordered relations on the criteria (weights) and the semantic meanings of the fuzzy concepts. Thus we can convert linguistic variables or fuzzy concepts in the dataset to exact numerical values through AFS membership function.

The rest of this paper is organized as follows: in the following section, we introduce the preliminaries of the AFS theory. Section 3 presents an approach to extend VIKOR method. In section 4, the proposed method is applied for supplier selection. Finally, conclusion is stated in Section 5.

\section{PRELIMINARIES OF THE AFS THEORY}

In this section, we recall some notations and properties of AFS theory that will be of immediate use in this study [4]. In essence, the AFS theory is based on AFS algebras-a kind of semantic methodology which is a family of completely distributivity lattices and AFS structuration-a kind of mathematical description of the data structure which is a special kind of combinatorics systems.

\subsection{Algebras AFS}

In order to study the semantics of expressions and the representations of fuzzy concepts, we need to present EI algebra-a kind of completely distributivity lattices, referred to as AFS algebras.

Theorem 1 [5]. Let $M$ be a non-empty set. The set $E M^{*}$ is defined as follows: $E M^{*}=\left\{\sum_{i \in I}\left(\prod_{m \in A i} m\right) \mid A_{i} \subseteq M, i \in I, I\right.$ is any non-empty indexing set $\}$.

$\left[\sum_{i \in I}\left(\prod_{m \in A i} m\right)\right] \sum\left[\vee_{i \in J}\left(\prod_{m \in B j} m\right)\right]=\sum_{k \in I} \cup_{J}\left(\prod_{m \in C k} m\right)$

$\left[\sum_{i \in I}\left(\prod_{m \in A i} m\right)\right] \sum\left[\wedge_{i \in J}\left(\prod_{m \in B j} m\right)\right]=\sum_{i \in I, j \in J}\left(\prod_{m \in A i \cup B j} m\right)$

In [5], the authors defined the logic operator ' (negation) as follows:

$\forall \sum_{i \in I} A_{i} \in E M, \quad\left({ }_{i \in I} A_{i}\right)^{\prime}=\wedge_{i \in I}\left(\vee_{a \in A}\left\{a^{\prime}\right\}\right)$.

If $m^{\prime}$ stands for the negation of fuzzy attribute $m \in M$, then for any fuzzy 
concept $\zeta \in E M, \zeta^{\prime}$ means the logical negation of $\zeta$. The algebra system ( $E M$, $\left.\vee, \wedge^{\prime}\right)$ is called AFS fuzzy logic.

\subsection{AFS Structures of the Data}

An AFS structure, a triple $(M, \tau, X)$, gives rise to various lattice representations of the membership degrees and fuzzy logic operations of the concepts in $E M$.

Definition $1[5,6]$. Let $X, M$ be sets and $2^{M}$ be the power set of $M$. Let $\tau: X \times X \rightarrow 2^{M}$. $(M, \tau, X)$ is called an AFS structure if $\tau$ satisfies the following axioms:

$$
\begin{aligned}
& \mathrm{AX} 1: \forall\left(x_{1}, x_{2}\right) \in X \times X, \tau\left(x_{1}, x_{2}\right) \subseteq \tau\left(x_{1}, x_{1}\right) \\
& \mathrm{AX} 2: \forall\left(x_{1}, x_{2}\right),\left(x_{2}, x_{3}\right) \in X \times X, \tau\left(x_{1}, x_{2}\right) \cap \tau\left(x_{2}, x_{3}\right) \subseteq \tau\left(x_{1}, x_{3}\right) .
\end{aligned}
$$

$X$ is called universe of discourse; $M$ is called a concept set and $\tau$ is called a structure.

In the real world applications, we can construct from the ordered relations on the attributes, let $X$ be a set of objects and $M$ be a set of simple concepts on $X$. If If $\tau: X \times X \rightarrow 2^{M}$ is defined as follows: for any $(x, y) \in X \times X$,

$$
\tau(x, y)=\left\{m \mid m \in M, x \geq_{m} y\right\}
$$

where $\geq_{m}$ is the ordered relation of the attribute $m \in M$. $x \geq_{m} y$ represents the degree of $x$ belonging to attribute $m$ is larger than or equal to that of $y$. Then $(M, \tau, X)$ is an AFS structure.

Definition 2 [5,7]. Let $X$ and $M$ be sets, $(M, \tau, X)$ be an AFS structure and $(X, \sigma, \mu)$ be a measure space, where $\mu$ is a finite and positive measure, $\mu(x) \neq 0$. If $A_{i}^{\tau}(x) \in \sigma$, for all $x \in X$, all $i \in I$, then for the fuzzy concept $\eta=\sum_{i \in I}$ $\left(\prod_{m \in A i} m\right) \in E M$, the membership function of $\eta$ is defined as follows: for any $x \in X$,

$$
\mu_{\eta}(x)=\sup _{i \in I} \frac{\sum_{u \in A_{i}^{\tau}(x)} \rho_{\gamma}(u) N_{u}}{\sum_{u \in X} \rho_{\gamma}(u) N_{u}}, \forall x \in X
$$

Where $A_{i}^{\tau}(x)=\left\{y \in X \mid \tau(x, y) \supseteq A_{i}\right\}=\left\{y \in X \mid x \geq_{m} y, \forall m \in A_{i}\right\}$.

In our study, let $\sigma=2^{X}$, for $W \in 2^{X}, m(W)=|W|(|W|$ is the cardinal number of the set $W$, i.e., the number of elements in $W$ ). Then (4) can be stated as follows:

$$
\mu_{\eta}(x)=\sup _{i \in I} \frac{\left|A_{i}^{\tau}(x)\right|}{|X|} .
$$

\section{EXTENDED VIKOR METHOD BASED ON AFS THEORY}

In real world, determining the exact values of some criteria (criteria weights) are difficult or impossible, it is more appropriate to express them not only by exact numerical values, but also by linguistic variables or fuzzy terms, so we try to extend VIKOR for the multi-type criteria (criteria weights) applying AFS theory. The merit of this approach is to convert linguistic variables or fuzzy terms to exact numerical values, then the VIKOR method can be used for more general MCDM problems.

Suppose $X_{1}, X_{2}, \ldots, X_{m}$ are $m$ possible alternatives among which decision-makers have to choose, $M_{1}, M_{2}, \ldots, M_{n}$ are criteria with which alternative performances are measured that $M_{1}, M_{2}, \ldots, M_{p}$ are represented through exact numerical values (membership degrees) and $M_{p+1}, M_{p+2}, \ldots, M_{n}$ through linguistic variables. A MCDM problem with linguistic variables can be expressed in matrix format (namely, the decision matrix) as:

$$
\begin{array}{cccccccc} 
& M_{1} & M_{2} & \cdots & M_{p+1} & M_{p+2} & \cdots & M_{n} \\
X_{1} & x_{11} & x_{12} & \cdots & x_{1 p} & l_{1(p+1)} & \cdots & l_{1 n} \\
X_{2} & x_{21} & x_{22} & \cdots & x_{2 p} & l_{2(p+1)} & \cdots & l_{2 n} \\
\vdots & \vdots & \vdots & \vdots & \vdots & \vdots & \vdots & \vdots \\
X_{m} & x_{m 1} & x_{m 2} & \cdots & x_{m p} & l_{m(p+1)} & \cdots & l_{m n} \\
& & & & & W=\left[w_{1}, w_{2}, \ldots, w_{n}\right]
\end{array}
$$

where $\omega_{j}$ is the weight of criteria $M_{j}$. Here the importance weights of various criteria are considered as linguistic variables. Thus $l_{i j}$ and $\omega_{j}$ are linguistic descriptions. The extending of the VIKOR method for the above problem is shown as follows:

a) For the criteria $M_{p+1}, M_{p+2}, \ldots, M_{n}$, on the basis of their respective linguistic descriptions, we have the ordered relations of all the alternatives on these criteria (refer to Section II.B). Then the AFS structure of the attributes $M_{p+1}, M_{p+2}, \ldots, M_{n}$ is established.

b) Calculate the membership degrees of each alternative on the criteria $M_{p+1}, M_{p+2}, \ldots, M_{n}$ according to Definition 2 so that the linguistic variables can be converted to exact numerical values.

c) Obtain the normalized weight. At first, we get the weight expressed by exact numerical value what like shown in the above steps, noted as : $W=\left[w_{1}, w_{2}, \ldots, w_{n}\right]$. Then, we translate the weight into $W=\left[\omega_{1}, \omega_{2}, \ldots, \omega_{n}\right]$ subjected to $\sum_{j=1}^{n} \omega_{j}=1$, computed $\quad$ as: $\omega_{j}=\tilde{w}_{j} / \sum_{j=1}^{n} w_{j} \quad$ (If $\quad$ the weight $W$ is expressed by numerical value, then this step is omitted)

d) Carry on the steps of VIKOR method.

\section{APPLICATION OF THE PROPOSED METHOD FOR SUPPLIER SELECTION}

Supply selection has received recently considerable attention in both academia and industry, which is extraordinarily important for the company to reduce production costs, maximize revenue, improve customer service, and resulting in increased competitiveness. Thus, the proposed model is applied for supplier selection problem to further demonstrate the application of the model.

An automotive company is desired to select the most appropriate supplier for one of the key elements in its manufacturing process. This problem considers 5 attributes and 6 alternative suppliers, which are evaluated by product quality $\left(a_{1}\right)$,production capacity ( $a_{2}$ ), financial condition $\left(a_{3}\right)$, product price ( $b_{1}$ ) and on-time delivery rate $\left(b_{2}\right)$,as presented in Table 1 ,which also includes the weights of each attribute. 
Table 1: Performance data of 6 suppliers

\begin{tabular}{|cccccc|}
\hline Suppliers & $a_{1}(0.2)$ & $a_{2}(0.3)$ & $a_{3}(0.3)$ & $b_{1}(0.1)$ & $b_{2}(0.1)$ \\
\hline$s_{1}$ & 0.81 & 0.97 & 230 & High & High \\
$s_{2}$ & 0.75 & 0.89 & 130 & High & High \\
$s_{3}$ & 0.73 & 0.80 & 160 & Low & High \\
$s_{4}$ & 0.84 & 0.86 & 180 & Medium & Low \\
$s_{5}$ & 0.74 & 0.87 & 150 & Very Low & Low \\
$s_{6}$ & 0.95 & 0.94 & 170 & Medium & Low \\
\hline
\end{tabular}

In what follows, we apply the proposed method to solve above issue.

a) Get the ordered relations on $b_{1}$ and $b_{2}$ by Table1.

b) Calculate the membership degrees of each supplier selection on $b_{1}$, $b_{2}$. By the formula (5) of Definition 2 , for $s_{1}$, we can get: A $=b_{1}, A^{\tau}$ $\left(s_{1}\right)=\left\{s_{1}, s_{2}, s_{3}, s_{4}, s_{5}, s_{6}\right\}, \mu_{c_{7}}\left(s_{1}\right)=\quad\left|A^{\tau}\left(s_{1}\right)\right| /|X|$ $=6 / 6=1$.

Similarly, we can also get the membership degrees of the other suppliers , and the results are shown as follows:

\begin{tabular}{|l|l|l|}
\hline & $b_{1}$ & $b_{2}$ \\
\hline$s_{1}$ & 1 & 1 \\
\hline$s_{2}$ & 1 & 1 \\
\hline$s_{3}$ & $1 / 3$ & 1 \\
\hline$s_{4}$ & $2 / 3$ & $1 / 2$ \\
\hline$s_{5}$ & $1 / 6$ & $1 / 2$ \\
\hline$s_{6}$ & $2 / 3$ & $1 / 2$ \\
\hline
\end{tabular}

c) Rank the alternatives, sorting by the values $S, R$, and Q in ascending order. Which are shown as follows (In this study, the weighting reference is 0.5):

$$
\begin{aligned}
& \text { S: } s_{1}<s_{6}<s_{4}<s_{2}<s_{3}<s_{5} \text {; } \\
& \text { R: } s_{1}<s_{2}=s_{3}=s_{4}=s_{5}=s_{6} \text { ； } \\
& \text { Q: } s_{1}<s_{6}<s_{4}<s_{2}<s_{3}<s_{5} \text {. }
\end{aligned}
$$

Obviously, $s_{1}$ is the best choice for the decision maker.

\section{CONCLUSION}

In this analysis, we applied the AFS theory to extend the VIKOR method used in fuzzy environment, and this means that we convert linguistic variables or fuzzy concepts in the dataset to exact numerical values through AFS membership function. Compared with other methods, the proposed method has the following advantages:
i.The types of criteria of the datasets can be mixed (numerical values, linguistic information or fuzzy terms),

ii.The fuzzy numbers and their distance function are not required.

The supplier selection is applied to verify the rationality and accuracy of the proposed method and it demonstrates the wide application of this method. The proposed method can also be applied to various MCDM problems.

\section{ACKNOWLEDGMENTS}

This work is supported by the Doctoral Scientific Research Foundation of LiaoNing Province, 201601286.

\section{ABOUT THE AUTHORS}

Lili Tao, PH.D., research direction is multi-attribute decision-making, lecturer in Dalian ocean university. Lishi Zhang, PH.D., research direction is applied mathematics, professor in Dalian ocean university. Lifeng Zhang, master, research direction is applied mathematics, associate professor in Dalian ocean university.

\section{REFERENCES}

[1] Zhenga, H., Xuana, G., Xiaoyua, S., Jua, L., Sheng, W.B. 2017. A coupled immersed boundary method for simulating multiphase flows. Acta Electronica Malaysia, 1 (1), 05-08.

[2] Lin, L., Lee, H.M. 2013. A new method of fuzzy decision making for diagnosing machine fault with compositional rule of inference. ICIC Express Letters, 7, 1865-1869.

[3] Chunxia, T. 2017. Research on the Multilevel Security Authorization Method Based on Image Content. Acta Electronica Malaysia, 1 (1), 8-20.

[4] Chaabene, M., Ben, A.M., Elhajjaji, A. 2007. Fuzzy approach for optimal energy management of a domestic photovoltaic panel. Appl Energy, 84, 992-1001.

[5] Liu, X.D. 1998. The fuzzy theory based on AFS algebras and AFS structure. Journal of Mathematical Analysis and Applications, 217, 459489.

[6] Liu, X.D. 2007.The development of AFS theory under probability theory. International Journal of Information and Systems Sciences, 3, 326348.

[7] Liu, X.D., Chai, T.Y., Wang, W. 2007. Approaches to the representations and logic operations for fuzzy concepts in the framework of axiomatic fuzzy set theory I, II. Information Sciences, 177, 1007-1045. 